\title{
The new phase of SEAJME
}

The South East Asian Journal of Medical Education (SEAJME) was initiated under the auspices of South East Asian Association for Medical Education (SEARAME) in the year 2007. This journal is the culmination of SEARAME's vision to bring together medical educationists in the region to promote collaborative efforts for uplifting the standards of medical education in the region and beyond through dissemination of knowledge and information.

The journal was initially published biannually from Chulalongkorn University, Bangkok, Thailand. The contribution of the Chulalongkorn secretariat in making SEAJME a medium for dissemination of good practices in medical education in the South East Asia region has been immense. In 2014, the editorial office of the journal was moved to the Faculty of Medicine, University of Colombo, Sri Lanka whilst the secretariat where publishing and dissemination of the journal takes place is in Coimbatore India. A new board of editors will be appointed by the Colombo editorial office.

SEAJME needs to move forward during the new phase of its development. There is a need to increase the frequency of publication of the journal. Plans have been discussed to make it a quarterly journal so that it can become Indexed, thereby attracting more research articles.

The field of medical education is going through a sea of changes with new methods replacing conventional teaching and learning practices. The South East Asian countries are also undergoing the waves of change. The future role of the journal will be to provide a forum where evidence of what is best for SEAR can be sought and documented and debated.

Dr. Indika Karunathilake

Editor-in-Chief, SEAJME 